\title{
The Immunohistochemical Expression of Matrix Metalloproteinases-2 and -9 in Low-Grade Appendiceal Mucinous Neoplasms: May These Neoplasms be Non-Invasive?
}

\author{
Matriks Metalloproteinaz-2 ve -9'in Düşük Dereceli Apendiks Müsinöz Neoplazmlarındaki Immünohistokimyasal \\ Ekspresyonu: Bu Neoplazmlar Invaziv Olmayabilir mi?
}

\section{Özgür Ekinci}

Gazi University, Faculty of Medicine, Department of Medical Pathology, Ankara, Turkey

\begin{abstract}
Objective: Low-grade appendiceal mucinous neoplasms (LAMN) are debated about their potential to cause invasion and pseudomyxoma peritoneii (PMxP). Our aim was to examine these neoplasms by two invasion markers: matrix metalloproteinases- 2 and -9 (MMP).

Methods: Twenty-eight cases of mucinous adenomas, LAMNs and adenocarcinomas were included. Immunohistochemistry of MMP-2 and -9 were applied and scored.

Results: Overall, the majority of LAMNs were negative by these two markers. Conclusion: LAMNs may not actually have an invasive nature but still they can cause PMxP. These patients should be followed.
\end{abstract}

Key Words: Appendix, mucinous neoplasm, matrix metalloproteinase
ÖZET

Amaç: Düşük dereceli apendiks müsinöz neoplazmlarının (DAMN) invazyona ve psödomiksoma peritoneii'ye (PMxP) neden olma potansiyelleri tartışmalıdır. Bu çalışmada amacımız, bu neoplazmları matriks metalloproteinaz-2 ve -9 invazyon belirteçleri ile incelemektir.

Yöntem: Müsinöz adenom, DAMN ve adenokarsinom tanısı almış 28 olgu dahil edildi. Matriks metalloproteinaz-2 ve -9 immünhistokimyasal boyamasları uygulandı ve ekspresyonları skorlandı.

Bulgular: Genel bakışta, DAMN'ların çoğunluğu bu belirteçler ile negatifti. Sonuç: DAMN'ler aslında invaziv tabiatta olmayabilir ancak yine de PMxP'ye neden olabilirler. Bu hastalar takip edilmelidir.

Anahtar Sözcükler: Appendiks, musinöz neoplazm, matriks metalloproteinaz

Kabul Tarihi: 17.02 .2018 


\section{INTRODUCTION}

The mucin-producing epithelial neoplasms of the appendix vermiformis with low-grade cytological atypia, only non-infiltrative expansion, partial or total atrophy of the normal mural layers replaced by a collagenous rim, excessive dilation of the lumen, probable presence of acellular mucin lakes dissecting the wall are all features of what is named a "low-grade mucinous appendiceal neoplasm (LAMN)" in several diagnostic classifications [World Health Organisation (WHO), American Joint Committee on Cancer (AJCC), Peritoneal Surface Oncology Group International (PSOGI)]. This neoplasm may be prone to cause pseudomyxoma peritoneii (PMxP), which can be fatal by causing intraabdominal mucin production and obstruction of visceral organs $(1,2,3)$.

We aimed to examine these tumors by their immunohistochemical expression for two markers of invasion, matrix metalloproteinase- 2 and -9 (MMP-2 and MMP-9), in order to evaluate their status of showing whether an invasive or non-invasive phenomenon. To the best of our knowledge, such a study was not performed previously.

\section{METHODS}

We used archival paraffin blocks of patients diagnosed with any primary mucinous neoplasm of the appendix vermiformis diagnosed in our institution between 2007 and 2014. Diagnoses included in the study were mucinous adenoma, low-grade mucinous neoplasm of the appendix, mucinous adenocarcinoma and signet ring cell carcinoma. Paraffin blocks that included the deepest invading portions of the tumors in the appendiceal wall were selected. Multiple numbers of blocks could be used.
Hematoxylin and eosin stained slides were evaluated for growth patterns (infiltrative vs expansile), cytological grade (low vs high), deepest level of invasion (mucosa, submucosa, muscularis propria, subserosa/mesoappendix or serosa), presence or absence of dissecting mucin lakes, if present, deepest level of the mucin lake and whether it contained neoplastic cells or was acellular. Presence of PMxP was recorded based on the examination of the surgical pathology specimen. Initial diagnoses were reviewed and renewed according to the recent recommendations $(1,2,3)$.

Immunohistochemistry for MMP-2 and MMP-9 were performed per recommendations of the manufacturers [Thermo Scientific ${ }^{\mathrm{TM}}$ MMP-2 (72kDa Collagenase IV) Ab-2, Mouse Monoclonal Antibody,and MMP-9 polyclonal antibody; Waltham, Massachusetts, ABD]. MMP-2 expression in the stroma around the tumoral cells and acellular mucin lakes were recorded separately. MMP-9 expression was evaluated in the tumor cell cytoplasm. Immunohistochemical results were semi-quantitatively scored based on the extent of positivity as 1,2 , or 3 . No staining meant score 0 .

Statistical analysis was performed with SPSS 15.0 software (SPSS Inc, Chicago, IL). Categorical variables were presented as frequencies and percentages. Categorical variables were compared using Chi-square test or Fisher's exact test. A P-value $<.05$ was considered to be statistically significant.

\section{RESULTS}

There were 35 patients. Seven of these were external consultation cases and paraffin blocks were not present in our archives. Consequently, we had 28 patients included in the study. Patients' ages were 29-79 (mean 60.27, median $66)$. There were 14 male and 14 female patients. A total of 72 paraffin blocks were used (mean 2.6, median 3). The original pathology diagnoses were mucinous adenoma (5 cases), low-grade mucinous neoplasm (11 cases) and mucinous adenocarcinoma (12 cases) (Table 1 ).

Table 1. Demographics and pathological features of the patients (M/F: male/female, I/E: invasive/expansile, L/H: low/high, M: mucosa, SM: submucosa, MP: muscularis propria, MAPP: mesoappendix/subserosa, P: visceral peritoneum/serosa, ATRP: total atrophy of the wall, Y/N: yes/no, PMxP: pseudomyxoma peritoneii and (-): not applicable).

\begin{tabular}{|c|c|c|c|c|c|c|c|c|c|}
\hline Patient & Gender & Age & $\begin{array}{l}\text { Number } \\
\text { of } \\
\text { blocks } \\
\text { used }\end{array}$ & $\begin{array}{l}\text { Growth } \\
\text { pattern }\end{array}$ & $\begin{array}{l}\text { Cytological } \\
\text { grade }\end{array}$ & $\begin{array}{l}\text { Depth } \\
\text { of } \\
\text { tumor } \\
\text { invasion }\end{array}$ & $\begin{array}{l}\text { Depth } \\
\text { of } \\
\text { mucin } \\
\text { invasion }\end{array}$ & $\begin{array}{l}\text { Presence } \\
\text { of tumor } \\
\text { cells in } \\
\text { mucin }\end{array}$ & PMxP \\
\hline 1 & $M$ & 53 & 4 & I & $\mathrm{H}$ & MAPP & MAPP & $Y$ & $\mathrm{~N}$ \\
\hline 2 & $M$ & 39 & 2 & $E$ & $\mathrm{~L}$ & SM & $P$ & $\mathrm{~N}$ & $\mathrm{~N}$ \\
\hline 3 & $\mathrm{~F}$ & 66 & 4 & 1 & $\mathrm{~L}$ & MAPP & MAPP & $Y$ & $\mathrm{~N}$ \\
\hline 4 & $M$ & 52 & 0 & $E$ & $\mathrm{~L}$ & $\mathrm{MP}$ & $(-)$ & $(-)$ & $\mathrm{N}$ \\
\hline 5 & $\mathrm{~F}$ & 77 & 3 & $E$ & $\mathrm{~L}$ & MAPP & MAPP & $Y$ & $\mathrm{~N}$ \\
\hline 6 & $M$ & 45 & 1 & 1 & $\mathrm{~L}$ & MAPP & $(-)$ & $(-)$ & $\mathrm{N}$ \\
\hline 7 & $\mathrm{~F}$ & 50 & 4 & $E$ & $\mathrm{~L}$ & ATRP & MAPP & $\mathrm{N}$ & $\mathrm{N}$ \\
\hline 8 & $M$ & 71 & 1 & $E$ & $L$ & ATRP & $(-)$ & $(-)$ & $\mathrm{N}$ \\
\hline 9 & $\mathrm{~F}$ & 68 & 4 & 1 & $\mathrm{H}$ & MAPP & MAPP & $Y$ & $\mathrm{~N}$ \\
\hline 10 & $\mathrm{~F}$ & 75 & 3 & $E$ & $\mathrm{~L}$ & ATRP & MAPP & $\mathrm{N}$ & $\mathrm{N}$ \\
\hline 11 & $M$ & 79 & 3 & 1 & $\mathrm{~L}$ & MAPP & MAPP & $\mathrm{N}$ & $\mathrm{N}$ \\
\hline 12 & $\mathrm{~F}$ & 66 & 3 & 1 & $\mathrm{~L}$ & $P$ & $P$ & $Y$ & $Y$ \\
\hline 13 & $\mathrm{~F}$ & 29 & 1 & $E$ & $\mathrm{~L}$ & $(-)$ & SM & $\mathrm{N}$ & $\mathrm{N}$ \\
\hline 14 & $\mathrm{~F}$ & 66 & 3 & 1 & $\mathrm{~L}$ & MAPP & MAPP & $Y$ & $\mathrm{~N}$ \\
\hline 15 & $M$ & 68 & 3 & $E$ & $\mathrm{~L}$ & $P$ & $P$ & $Y$ & $Y$ \\
\hline 16 & $M$ & 70 & 3 & 1 & $\mathrm{~L}$ & $P$ & $P$ & $Y$ & $Y$ \\
\hline 17 & $M$ & 63 & 3 & $E$ & $\mathrm{~L}$ & $M$ & SM & $(-)$ & $\mathrm{N}$ \\
\hline 18 & $\mathrm{~F}$ & 50 & 2 & I & $\mathrm{L}$ & $P$ & $P$ & $\mathrm{Y}$ & $\mathrm{N}$ \\
\hline 19 & $M$ & 55 & 1 & $E$ & $\mathrm{~L}$ & $M$ & $(-)$ & $(-)$ & $\mathrm{N}$ \\
\hline 20 & $\mathrm{~K}$ & 42 & 2 & 1 & $\mathrm{H}$ & $P$ & $P$ & $Y$ & $\mathrm{~N}$ \\
\hline 21 & $\mathrm{~F}$ & 71 & 3 & $E$ & $\mathrm{~L}$ & $M$ & SM & $\mathrm{N}$ & $\mathrm{N}$ \\
\hline 22 & $M$ & 58 & 4 & $E$ & $\mathrm{~L}$ & ATRP & MAPP & $\mathrm{N}$ & $\mathrm{N}$ \\
\hline 23 & $\mathrm{~F}$ & 66 & 3 & $E$ & $L$ & $\mathrm{MP}$ & $\mathrm{MP}$ & $\mathrm{N}$ & $\mathrm{N}$ \\
\hline 24 & $\mathrm{M}$ & 59 & 5 & $E$ & $L$ & ATRP & $\mathrm{MP}$ & $\mathrm{N}$ & $\mathrm{N}$ \\
\hline 25 & $\mathrm{~F}$ & 77 & 2 & $E$ & $\mathrm{~L}$ & ATRP & MAPP & $\mathrm{N}$ & $\mathrm{N}$ \\
\hline 26 & $M$ & 54 & 2 & 1 & $\mathrm{~L}$ & MAPP & MAPP & $Y$ & $\mathrm{~N}$ \\
\hline 27 & $\mathrm{~F}$ & 34 & 2 & $E$ & $\mathrm{~L}$ & MAPP & $\mathrm{P}$ & $\mathrm{N}$ & $\mathrm{N}$ \\
\hline 28 & $M$ & 79 & 3 & $E$ & $\mathrm{~L}$ & MAPP & MAPP & $Y$ & $\mathrm{~N}$ \\
\hline
\end{tabular}


When the original diagnoses were renewed according to WHO 2010 and AJCC 2018 criteria, the following alterations of the diagnoses were made: i) in 11 cases discordance was present among the original and WHO, AJCC and PSOGI diagnoses; ii) in 2 cases where the diagnoses were LAMN, the AJCC diagnosis was "LAMN - pT4a"; iii) in another 2 cases, the original and WHO diagnosis of a "well-differentiated mucinous adenocarcinoma" was to be substituted for a "LAMN, pT4a" diagnosis; iv) for 3 cases, the diagnosis of "mucinous adenoma" was replaced with LAMN according to WHO, AJCC and PSOGI criteria; v) in 3 cases, while both the initial report and the application of WHO criteria yielded a diagnosis of LAMN, based on the AJCC recommendations this focus should be called "LAMN-pT3"; vi) one mucinous adenoma that was also a mucinous adenoma for WHO and AJCC schemes was changed to LAMN based on the PSOGI criteria. In the WHO and AJCC schemes, 3 cases of MA were upgraded to LAMN. The AJCC approach altered all LAMN cases to Tis, T3 or T4a and 2 cases of WAdCa to LAMN-T4a. The PSOGI method converted all MA cases and 1 WAdCa case to LAMNs (Table 2).

Table 2. The documentation of the expressions of MMP-2 and -9 and the diagnoses according to the three systems, preceded by the original diagnosis. MA: mucinous adenoma; LAMN: low-grade appendiceal mucinous neoplasm; Tis-LAMN: Tumor In Situ, low-grade appendiceal mucinous neoplasm; WAdCa, MAdCa, PAdCa: well-, moderately- and poorly-differentiated adenocarcinoma, respectively. For the description of LAMN-T3 and LAMN-T4a see text.

\begin{tabular}{|c|c|c|c|c|c|c|c|}
\hline Patient & $\begin{array}{l}\text { MMP-2 } \\
\text { around } \\
\text { tumor }\end{array}$ & $\begin{array}{l}\text { MMP-2 } \\
\text { around } \\
\text { acellular } \\
\text { mucin }\end{array}$ & $\begin{array}{l}\text { MMP-9 } \\
\text { in tumor } \\
\text { cells }\end{array}$ & $\begin{array}{l}\text { Original } \\
\text { diagnosis }\end{array}$ & $\begin{array}{l}\text { WHO } 2010 \\
\text { Diagnosis }\end{array}$ & $\begin{array}{l}\text { AJCC } 2017 \\
\text { Diagnosis }\end{array}$ & $\begin{array}{l}\text { PSOGI } \\
2017 \\
\text { Diagnosis } \\
\end{array}$ \\
\hline 1 & 0 & $(-)$ & 0 & MA & LAMN & Tis-LAMN & LAMN \\
\hline 2 & 0 & 0 & 0 & MA & LAMN & Tis-LAMN & LAMN \\
\hline 3 & 0 & $(-)$ & 0 & MA & $\mathrm{MA}$ & $\mathrm{MA}$ & LAMN \\
\hline 4 & 0 & 0 & 0 & MA & LAMN & Tis-LAMN & LAMN \\
\hline 5 & 0 & 2 & 0 & LAMN & LAMN & LAMN-T4a & LAMN \\
\hline 6 & 1 & $(-)$ & 0 & LAMN & LAMN & Tis-LAMN & LAMN \\
\hline 7 & 0 & 0 & 0 & LAMN & LAMN & LAMN-T3 & LAMN \\
\hline 8 & 0 & 0 & 0 & LAMN & LAMN & Tis-LAMN & LAMN \\
\hline 9 & 0 & 0 & 0 & LAMN & LAMN & Tis-LAMN & LAMN \\
\hline 10 & 0 & 0 & 0 & LAMN & LAMN & LAMN-T3 & LAMN \\
\hline 11 & 0 & 0 & 0 & LAMN & LAMN & Tis-LAMN & LAMN \\
\hline 12 & 0 & 1 & 0 & LAMN & LAMN & Tis-LAMN & LAMN \\
\hline 13 & 0 & 0 & 0 & LAMN & LAMN & LAMN-T3 & LAMN \\
\hline 14 & 0 & 0 & 0 & LAMN & LAMN & LAMN-T4a & LAMN \\
\hline 15 & 0 & 0 & 0 & LAMN & LAMN & LAMN-T3 & LAMN \\
\hline 16 & 2 & 3 & 0 & WadCa & WAdCa & LAMN-T4a & LAMN \\
\hline 17 & 1 & $(-)$ & 0 & WadCa & WAdCa & WAdCa & WAdCa \\
\hline 18 & 1 & 1 & 2 & WadCa & WAdCa & WAdCa & WAdCa \\
\hline 19 & 0 & $(-)$ & 0 & WadCa & WAdCa & WAdCa & WAdCa \\
\hline 20 & 0 & 1 & 0 & WadCa & WAdCa & WAdCa & WAdCa \\
\hline 21 & 0 & 0 & 2 & WadCa & WAdCa & LAMN-T4a & WAdCa \\
\hline 22 & 1 & 2 & 0 & WadCa & WAdCa & WAdCa & WAdCa \\
\hline 23 & 0 & 0 & 0 & WadCa & WAdCa & WAdCa & WAdCa \\
\hline 24 & 2 & 2 & 0 & WadCa & WAdCa & WAdCa & WAdCa \\
\hline 25 & 0 & 0 & 0 & WadCa & WAdCa & WAdCa & WAdCa \\
\hline 26 & 2 & 1 & 0 & WadCa & WAdCa & WAdCa & WAdCa \\
\hline 27 & 3 & 1 & 1 & MadCa & MAdCa & MAdCa & MAdCa \\
\hline 28 & 2 & 3 & 2 & PadCa & PAdCa & PAdCa & PAdCa \\
\hline
\end{tabular}

The expression of MMP-2 in the stroma around tumor cells showed these results: for the original, WHO and AJCC-based diagnoses, no staining was present in mucinous adenomas $(p=0.004$ and $p=0.014$ for the latter two approaches, respectively). For the original diagnosis, LAMNs and adenocarcinomas were positive in $1(9 \%)$ case and $8(61 \%)$ cases, respectively $(p=0.13)$. When the WHO 2010 criteria were used, 1 (7\%) LAMN and $8(61 \%)$ of adenocarcinomas were positive $(p=0.004)$. Based on the AJCC criteria, 2 $(13 \%)$ of LAMNs and $7(63 \%)$ of adenocarcinomas were positive $(p=0.014)$. For the PSOGI criteria, MMP-2 was positive in $2(12 \%)$ and $8(61 \%)$ in LAMNs and adenocarcinomas, respectively ( $p=0.016$; see Table 3 ). 
Table 3. The documentation of the expression of MMP-2 and -9 of the cases based on the original diagnoses and the three other classification systems. MA: mucinous adenoma; LAMN: low-grade appendiceal mucinous neoplasm; AdCa: adenocarcinoma. The AJCC diagnosis of Tis-LAMN is integrated into LAMN; all adenocarcinomas are integrated into $\mathrm{AdCa}$. The statistically significant $\mathrm{p}$ values are shown in bold.

\begin{tabular}{lllll}
\hline & MA & LAMN & AdCa & P-value \\
\hline Original Diagnosis & & & & \\
MMP-2 positive around tumor & $0 / 4$ & $1 / 11(9 \%)$ & $8 / 13(61 \%)$ & $\mathbf{0 . 0 1 3}$ \\
MMP-2 positive around acellular mucin & $0 / 4$ & $2 / 11(18 \%)$ & $8 / 13(61 \%)$ & $\mathbf{0 . 0 4 7}$ \\
MMP-9 positive in tumor cells & $0 / 4$ & $0 / 11$ & $4 / 13(30 \%)$ &
\end{tabular}

\section{WHO 2010 Diagnosis}

MMP-2 positive around tumor

MMP-2 positive around acellular mucin

$0 / 1$

$1 / 14(7 \%)$

$8 / 13(61 \%)$

MMP-9 positive in tumor cells

$0 / 1$

$2 / 14(14 \%)$

$8 / 13(61 \%)$

$0 / 1$

$0 / 14$

$4 / 13(30 \%)$

AJCC 2017 Diagnosis

\begin{abstract}
MMP-2 positive around tumor
\end{abstract}
$0 / 1$

2/15 (13\%) 7/11 (63\%)

MMP-2 positive around acellular mucin

$0 / 1$

$3 / 15(20 \%)$

$7 / 11(63 \%)$

MMP-9 positive in tumor cells

$0 / 1$

$1 / 15(6 \%) \quad 3 / 11(27 \%)$

PSOGI 2017 Diagnosis (This scheme does not include "mucinous adenoma", see text)
MMP-2 positive around tumor

MMP-2 positive around acellular mucin

MMP-9 positive in tumor cells

$\begin{array}{ll}2 / 16(12 \%) & 8 / 13(61 \%) \\ 3 / 16(18 \%) & 8 / 13(61 \%) \\ 0 / 16 & 4 / 13(30 \%)\end{array}$

$0 / 16$

$4 / 13(30 \%)$

\subsection{4}

0.018

0.041

0.014

0.043

0.279
0.027
MMP-2 in the stroma around acellular mucin lakes revealed the following results: for the original, $\mathrm{AJCC}$ and WHO-based diagnoses, no staining was present in mucinous adenomas. According to the original diagnosis, $2(18 \%)$ of LAMNs and $8(\% 61)$ of adenocarcinomas were positive $(p=0.047)$. When WHO 2010 criteria were used, 2 (14\%) of LAMNs and $8(61 \%)$ of adenocarcinomas were positive $(p=0.018)$. Usage of AJCC criteria revealed that $3(20 \%)$ and 7 $(63 \%)$ of cases of LAMNs and adenocarcinomas were positive, respectively $(p=0.043)$. When PSOGI criteria were applied, there was positivity in $3(18 \%)$ and $8(61 \%)$ in LAMNs and adenocarcinomas, respectively $(p=0.027$; see Table 3).

The expression of MMP-9 in the tumor cells' cytoplasm was recorded by the four diagnostic methods as well. Based on the original and WHO diagnoses, only the adenocarcinomas were positive in $4(30 \%)$ and $4(30 \%)$ of the cases, respectively (not applicable for the former and $p=0.041$ for the latter comparison). Based on the AJCC scheme, 1 (6\%) LAMN and $3(27 \%)$ adenocarcinomas were positive $(p=0.279)$. According to the PSOGI criteria, only $4(30 \%)$ adenocarcinoma cases were positive ( $p$ value not applicable; see Table 3).

PMxP was present in 3 cases of WAdCa. Two were WAdCa in the original report and by the other three criteria, while 1 case was diagnosed as LAMNT4a by the AJCC criteria. This latter case showed low-grade cytology and an expansile growth pattern (patient 15).

\section{DISCUSSION}

The 2010 WHO recommendations included LAMN under the heading of adenocarcinoma of the appendix, along with mucinous adenocarcinoma and signet ring cell carcinoma (1). They defined LAMN as "a tumor with broad front invasion characterized by atrophy and fibrosis of the underlying submucosa and muscularis propria and cells with low grade dysplasia". Recently, AJCC eight edition of 2017 has incorporated a Tis(LAMN) category reserved for LAMNs that are "confined by the muscularis propria" and may have " acellular mucin or mucinous epithelium invading into the muscularis propria" (2). In addition, pT1 and pT2 (that is, submucosal and muscularis propria invasion, respectively) stages are not applicable to LAMN in this scheme. If a LAMN should invade beyond the muscularis propria, the tumor is to be a LAMN-pT3 if it penetrates into the subserosa/mesoappendix, or a LAMN-pT4 if it reaches the peritoneum (serosa, pT4a) or invades an adjacent organ (pT4b).

These classification proposals have differences in diagnostic and oncological staging terms. While WHO recognizes the atrophy of the mural layers and development of fibrosis as a diagnostic feature of LAMNs, AJCC does not address this issue and it is not clear how to diagnose or stage these LAMNs by AJCC criteria. For this matter, PSOGI recommendations are dissimilar. They emphasize that a LAMN may cause two types of extension in the wall: first is the pushing invasion characterized by tongue-like or diverticulum-like protrusions or broad-front spread of epithelium, second is the dissection of acellular mucin in the wall. According to their group's consensus criteria, LAMN is a mucinous neoplasm without infiltrative invasion but with any of the following (in addition to the two features described above): loss of muscularis mucosae, fibrosis of submucosa, rupture of the appendix, mucin and/or cells outside the appendix. They reject the diagnosis of "mucinous adenoma" and "prefer to limit the usage of 'adenoma' to lesions that resemble tubular, tubulovillous or villous adenoma of the colorectum", and recommend the diagnoses of LAMN or HAMN (high-grade AMN) for these lesions. They base this decision on their experience that even the mucinous tumors confined to the muscularis mucosae might cause pseudomyxoma peritoneii (3).

This ongoing debate appears to be a reflection of the inconsistency between the grade and stage in cases of low grade and even low stage mucinous neoplasms that may synchronously or metachronously be associated with pseudomyxoma peritoneii. In this context, our aim was to examine these low grade tumors for their profile of two invasion-related proteins, MMP-2 and MMP-9, in order, if possible, to characterize or classify these neoplasms, as their $\mathrm{H} \& \mathrm{E}$ microscopic appearance may be inadequate to stratify them for their expected biological behavior.

Matrix metalloproteinases are known for their involvement in the destruction of the extra-cellular matrix elements and neoplastic invasion (4, 5, 6). Our method using the two MMP's, 2 and 9, in appendiceal mucinous neoplasms was not performed in the past, to the best of our knowledge. As documented above, the nature of these tumors require a comprehensive investigation. Our method was to test LAMNs against appendiceal adenocarcinomas for the positivity of MMP-2 and -9 . Our hypothesis was that these low grade neoplasms might not actually be invasive tumors.

The expression of these two markers was significantly lower in LAMNs compared to the adenocarcinomas according to all four diagnostic parameters: the original reports, and WHO, AJCC and PSOGI criteria. On the other hand, some LAMNs did stain positively for these MMPs, though in a low ratio: 3 out of 11 were positive for MMP-2 (when the two staining results were combined), 3 out of 14 was positive for MMP-2, 5 out of 15 were positive for MMP-2 according to the original, WHO, AJCC and PSOGI diagnoses, respectively. MMP-9 was positive in 1 case $(6 \%)$ only based on the AJCC scheme, while there was no positivity for this marker in the original diagnosis, and the WHO and PSOGI systems. The positivity of this 1 case for MMP-9 was not statistically significant. While these results may indicate a discrepancy, still, the majority of LAMNs were negative for these invasion markers according to all diagnostic schemes.

The original diagnoses were to be altered in several cases. The minimum amount of cases that gained another diagnosis was for the WHO criteria where 3 cases of MAs were upgraded to LAMNs. This was followed by the PSOGI method, where all of the 4 MAs were re-diagnosed as LAMNs and 1 case of WAdCa was changed to a LAMN. By its novelty, the recent AJCC system brought about alterations in 15 cases of LAMNs and a case of WAdCa. 
Overall, upgrading took place for 3 MAs in WHO, AJCC and 4 MAs in PSOGI schemes. Whether the novel nomenclature of AJCC's approach for rediagnosing 2 WAdCa's as LAMN-T4a's signifies a downgrading of the diagnosis or pertains a prognostic significance was not in the scope of this study and should better be reserved for future studies with follow-up.

We accept that the mere positivity of LAMNs by one or both of these two MMPs may not be considered as an absolute indicator of an inherent invasive nature. Nevertheless, the statistically significant negativity of LAMNs for these two markers may be a signifier that the majority of these cases are actually of a non-invasive biology, and they may cause rupture and spilling-out of mucinproducing neoplastic cells throughout the peritoneal cavity, which can yield to PMxP.

As we have not observed any MMP positivity in MAs from the original or other two diagnostic series, we are not sure of the PSOGI recommendations that propose that a "mucinous adenoma" of appendix vermiformis does not exist and these are actually LAMNs. We can propose that, considering the positivity of any of the two MPPs used in this study, LAMNs cannot definitely be diagnosed by pathological examination only. We recommend that all patients with this diagnosis should be closely followed.

In conclusion, our findings present that, even if most LAMNs may exhibit a non-invasive phenotype, at least a minority of these tumors may actually be invasive. Our findings should be correlated to the prognosis and follow-up of these patients in new researches.
Conflict of interest

No conflict of interest was declared by the authors.

\section{REFERENCES}

1. Bosman FT, Carnerio F, Hruban RH, Theise ND (Eds): WHO Classification of Tumours of the Digestive System. 4th ed. IARC: Lyon 2010.

2. Amin MB, Edge SB, Grene FL, Byrd DR, Brookland RK, Washington MK Gershenwald JE, Compton CC, Hess KR, Sullivan DC, Jessup JM, Brierley JD, Gaspar LE, Schilsky RL, Balch CM, Winchester DP, Asare EA, Madera M, Gress DM, Meyer LR. AJCC Cancer Staging Manual. 8th ed. Springer; 2017.

3. Carr NJ, Bibeau F, Bradley RF, Dartigues P, Feakins RM, Geisinger KR, et al. The histopathological classification, diagnosis and differential diagnosis of mucinous appendiceal neoplasms, appendiceal adenocarcinomas and pseudomyxoma peritonei. Histopathology 2017; 71: 847-58.

4. Westermarck J, Kähäri VM. Regulation of matrix metalloproteinase expression in tumor invasion. FASEB J 1999; 13: 781-92.

5. Seiki M. Membrane-type 1 matrix metalloproteinase: a key enzyme for tumor invasion. Cancer Lett 2003; 194: 1-11.

6. Cockett MI, Birch ML, Murphy G, Hart IR, Docherty AJ. Metalloproteinase domain structure, cellular invasion and metastasis. Biochem Soc Trans 1994; 22: 55-7. 